\title{
Control of Drug Dissolution Rate from Film Dosage Forms Containing Valsartan
}

\author{
Yoshifumi Murata, Kyoko Kofuji, and Chieko Maida \\ Faculty of Pharmaceutical Science, Hokuriku University, Ho-3, Kanagawa-machi, Kanazawa 920-1181, Japan \\ Correspondence should be addressed to Yoshifumi Murata; y-murata@hokuriku-u.ac.jp
}

Received 29 February 2016; Revised 13 April 2016; Accepted 4 May 2016

Academic Editor: Giuseppe Biondi-Zoccai

Copyright ( 2016 Yoshifumi Murata et al. This is an open access article distributed under the Creative Commons Attribution License, which permits unrestricted use, distribution, and reproduction in any medium, provided the original work is properly cited.

Film dosage forms (FDs) containing valsartan (VST), a popular antihypertensive drug, were prepared using a casting method with sodium alginate and other polysaccharides as the film base. Drug dissolution profiles of the FDs were investigated in limited medium. The FDs were 170-200 $\mu \mathrm{m}$ thick and were easy to handle. All FDs immediately swelled and disintegrated in the medium. About $23 \%$ of the VST incorporated into the FD prepared with $1.5 \%$ sodium alginate dissolved at $5 \mathrm{~min}$. The initial dissolution rate of VST increased upon the addition of chitosan to the film base; this effect was not observed in the case of chitin. On the other hand, the rate apparently decreased upon modification with alginic acid. In addition, the solubility of VST in the dissolution medium was changed by the addition of chitosan or alginic acid. FDs prepared with polysaccharides are useful for simplifying the administration of drugs to patients, and the drug dissolution rate from FDs can be controlled by modification.

\section{Introduction}

Film has been noted as an excellent dosage form, especially in oral care. When a film dosage form (FD) is set in a small amount of liquid, it swells quickly and releases the compounds incorporated in the film matrix [1]. FDs can be attached in the oral cavity, allowing drug distribution across the membrane $[2,3]$. This can be useful for patients who have difficulty swallowing regular oral dosage forms, such as tablets or capsules [4-6]. However, the utilization of film as a dosage form is limited because the drug loading capacity is low. Furthermore, the drug dissolution profile is typically dependent on the properties of the drug, such as solubility in an aqueous medium. Therefore, control of the drug dissolution rate from the FD is difficult.

It is known that thin films can be prepared using a natural polysaccharide, such as sodium alginate (Alg-Na) or pullulan, without dissolution in organic solvents, heating, or $\mathrm{pH}$ regulation. We have already reported the characteristics of FDs prepared with Alg-Na, which has been used widely as a food additive, a tablet disintegrator, or a gelation agent [7]. Moreover, Alg-Na itself is used to treat ulcers by protecting the gastric mucosa.
When preparing the film using the casting method, FD is formed by the evaporation of solvent from the film base solution containing an active compound. FDs prepared with Alg-Na can be modified by additives within the limits of film formation. For example, the drug dissolution rate of miconazole was promoted by the addition of a cyclodextrin to the film base solution [8]. In this study, valsartan (VST), an angiotensin II receptor blocker, was selected as the model drug to be incorporated into an FD [9]. We attempted to modify the FD using alginic acid (ALG) or chitosan (CS) as additives. The drug release profiles from the FDs were also determined in a limited dissolution medium.

\section{Materials and Methods}

2.1. Materials. Alg-Na (300 cps) was obtained from Nacalai Tesque Inc. (Kyoto, Japan). Low-molecular-weight Alg-Na (80-120 cps), ALG-S (swelling type), ALG-NS (nonswelling type), and the model drug VST were obtained from Wako Pure Chemicals (Osaka, Japan). Chitosan (CS; degree of deacetylation (DA) 75-85\%), CS-F, was obtained from Kimitsu Chemical Industries Co. Ltd. (Tokyo, Japan). CS-K (DA 
$85 \%$ ) was obtained from Koyo Chemical Co. (Osaka, Japan) and $\beta$-CS (DA 96\%) from Yaegaki Bio-Industry Inc. (Himeji, Japan). Chitin was obtained from Nacalai Tesque Inc. Pullulan was supplied by Hayashibara Biochemical Laboratories (Okayama, Japan). All other chemicals were of reagent grade.

2.2. FD Preparation. Alg-Na $(1.5 \%[\mathrm{w} / \mathrm{w}])$ containing an additive was prepared in deionized water as the film base solution. VST $(50 \mathrm{mg}$ ) was added with agitation to $10 \mathrm{~g}$ of the film base solution. The mixture was thoroughly mixed by sonication, and then $3.0 \mathrm{~g}$ of each solution was poured into individual plastic $54 \mathrm{~mm}$ Petri dishes. After $24 \mathrm{~h}$ at $37^{\circ} \mathrm{C}$, the circular films formed on each dish were transferred to a desiccator. Film formation was judged to have failed if a circular film was not obtained, if the film had cracks, or if the film could not be removed from the bottom of the dish. In the present method, $15 \mathrm{mg}$ of VST was theoretically incorporated into each film dosage form.

2.3. Film Thickness and Rheological Properties. Thickness was measured at 10 points on each film using a micrometer (CLM1-15QM; Mitutoyo, Kawasaki, Japan) with a set pressure of $0.5 \mathrm{~N}$. Measurements were made using 3 films, and the mean thickness was calculated for each type. The rheological properties of each film were determined using a rheometer (SUN RHEO TEX SD-700\#; Sun Scientific Co., Tokyo, Japan) at room temperature. The film was fixed on a vial (inner diameter $1.4 \mathrm{~mm}$, outer diameter $18.8 \mathrm{~mm}$ ) using a rubber band (Kyowa Co., Osaka) and was probed with a cylindrical adapter (diameter $5.0 \mathrm{~mm}$ ). Stress and strain were measured at the point at which the adapter broke through the film. The tests were performed in triplicate.

2.4. Solubility of VST. The solubility of VST was measured in physiological saline containing ALG-S, CS, or chitin. VST $(10 \mathrm{mg})$ and an additive $(10 \mathrm{mg}$ or $20 \mathrm{mg})$ were added to $20 \mathrm{~mL}$ of the test solution and shaken at $37^{\circ} \mathrm{C}$ for $24 \mathrm{~h}$; then, the suspension was removed using a preheated plastic syringe (Terumo Co., Tokyo) at $37^{\circ} \mathrm{C}$ and filtered using a syringe driven filter unit (Millex-HV, pore size: $0.45 \mu \mathrm{m}$, Millipore Co., MA, USA). The solution was diluted with methanol and injected onto an HPLC column.

2.5. Determination of VST. The HPLC system (Hitachi Co., Tokyo) consisted of a pump (L-2130), UV-detector (L2400), autosampler (L-2200), and chromate-integrator (D$2500)$ connected to a packed column $(150 \mathrm{~mm} \times 4.6 \mathrm{~mm}$, Cosmosil 5C $\mathrm{C}_{18}$-MS-II, Nacalai Tesque Inc.). To determine the concentration of VST, HPLC was conducted at ambient temperature using an eluent consisting of $10 \mathrm{mM}$ phosphate buffer ( $\mathrm{pH}$ 3.0), methanol, and acetonitrile $(19: 26: 5)$ at a flow rate of $1.0 \mathrm{~mL} / \mathrm{min}[10]$. The detector wavelength was set at $230 \mathrm{~nm}$.

2.6. VST Dissolution Test. An FD was placed in a plastic dish and $10 \mathrm{~mL}$ of the dissolution medium (physiological saline preheated to $37^{\circ} \mathrm{C}$ ) was added. The dish was shaken at $300 \mathrm{rpm}$ in a shaker incubator (SI-300; As One Co., Osaka,
TABLE 1: Thicknesses of FDs prepared with 1.5\% Alg-Na containing $0.5 \%$ additive.

\begin{tabular}{lc}
\hline Additive & Thickness $(\mu \mathrm{m})$ \\
\hline- & $125 \pm 2$ \\
ALG-S & $203 \pm 14$ \\
ALG-NS & $198 \pm 12$ \\
CS-F & $180 \pm 7$ \\
CS-K & $174 \pm 12$ \\
$\beta-C S$ & $166 \pm 7$ \\
Chitin & $174 \pm 2$ \\
\hline
\end{tabular}

Japan) at $37^{\circ} \mathrm{C}$. After $1,3,5,10,15,20,30,45$, and 60 minutes, a $0.3 \mathrm{~mL}$ aliquot of each solution was removed periodically using a plastic syringe, after which $0.3 \mathrm{~mL}$ of the test medium $\left(37^{\circ} \mathrm{C}\right)$ was added to maintain a constant volume. The solution was filtered through a syringe driven filter unit (pore size: $0.45 \mu \mathrm{m}$ ). Then, $80 \mu \mathrm{L}$ aliquots of the filtered solution were placed into micro-test-tubes $(1.5 \mathrm{~mL})$ and $720 \mu \mathrm{L}$ of methanol was added to precipitate the polysaccharide dissolved from the dosage form. Samples were mixed and centrifuged (7,700 ×g, 5 min; H-1300; Kokusan Co., Saitama, Japan), and the supernatants were injected into the HPLC column. All tests were performed in triplicate.

\section{Results and Discussion}

Using the casting method, an aqueous solution of Alg- $\mathrm{Na}$ or pullulan was poured into a Petri dish, and a thin film was formed after evaporation of the solvent. The addition of a drug to the base solution interfered with film formation depending on the polysaccharide. When $0.5 \%$ VST was added to a $1.5 \%$ Alg-Na solution, a $125 \mu \mathrm{m}$ thick circular film was obtained with VST homogeneously dispersed. However, pullulan (4-6\%) did not form an FD when it contained the same amount of VST. An additive such as ALG-S was added to the film base solution in order to show that the amount of additive also affects FD preparation. As shown in Figure 1, FDs were obtained from the Alg-Na solution containing $0.5 \%$ ALG-S or $0.5 \%$ ALG-NS, though the films prepared with the solution containing $1 \%$ ALG-S, $1 \%$ ALG-NS, or $1 \%$ CS were cracked. In the case of the $1.5 \%$ Alg-Na solution containing each additive, the thickness of the FDs was $170-200 \mu \mathrm{m}$, as shown in Table 1.

Because FDs containing VST are used for oral administration, they must be easy to handle as the forms are applied to the oral cavity one by one [11]. Table 2 shows the effect that additives have on the rheological properties of FDs prepared with Alg-Na. All FDs have enough strength to be manipulated by hand. The strength of the FD prepared with $1.5 \%$ Alg- $\mathrm{Na}$ containing $0.5 \%$ chitin could not be measured because of its fragility.

FDs prepared with a water-soluble polysaccharide immediately swell in physiological saline and then release the drug as they dissolve. Figure 2 shows the dissolution profiles of VST from FDs prepared with $1.5 \% \mathrm{Alg}-\mathrm{Na}$. When no additive was used, $3.5 \pm 0.2 \mathrm{mg}(23 \pm 1 \%)$ of VST was released from 


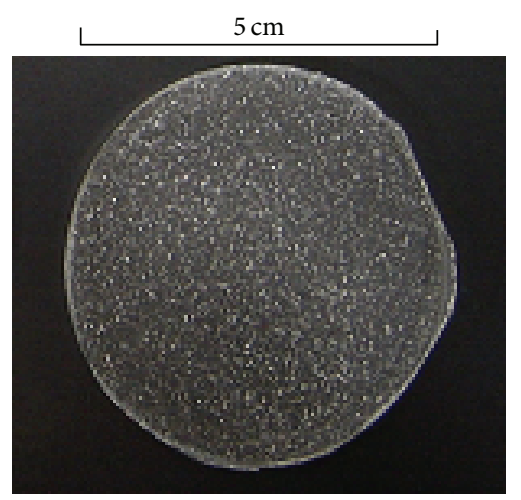

(a)

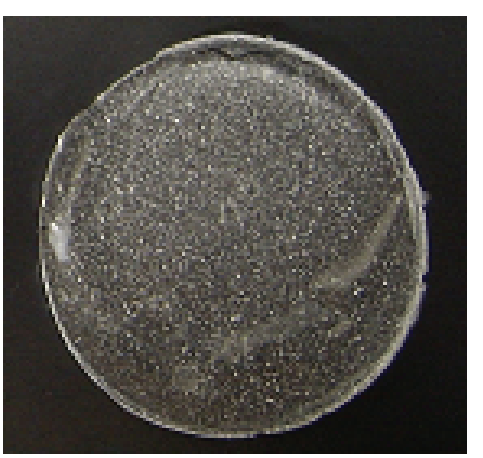

(b)

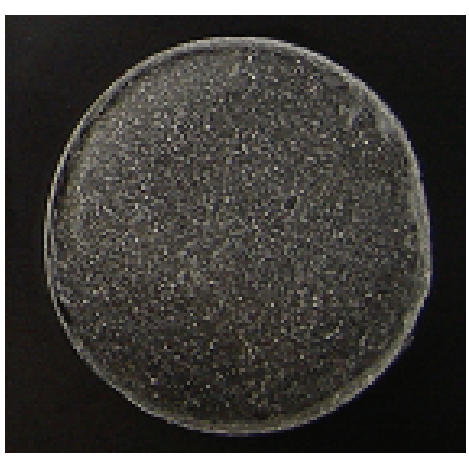

(c)

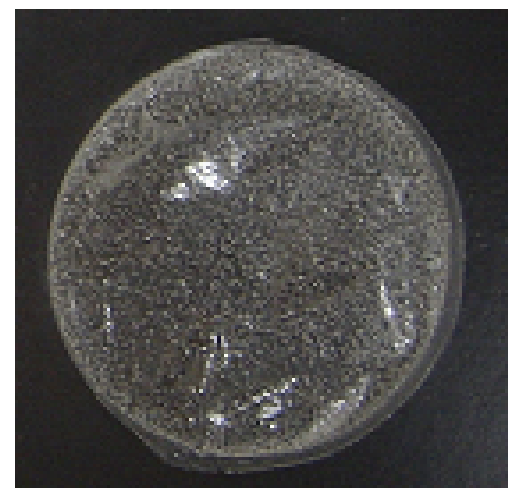

(d)

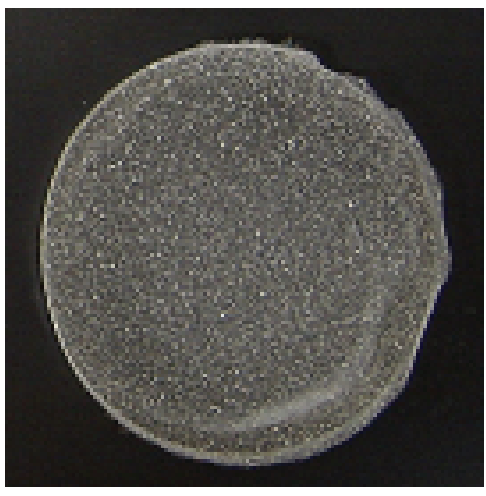

(e)

FIGURE 1: Pictures of FDs prepared with 1.5\% Alg-Na and 0.5\% additive containing VST: (a) additive-free; (b) ALG-S; (c) ALG-NS; (d) CS-F; (e) chitin.

TABLE 2: Rheological properties of FDs incorporating VST prepared with $1.5 \%$ Alg-Na containing $0.5 \%$ additive.

\begin{tabular}{lcc}
\hline Additive & Stress $(\mathrm{kPa})$ & Strain $(\mathrm{mm})$ \\
\hline- & $151 \pm 6$ & $2.3 \pm 0.3$ \\
ALG-S & $206 \pm 44$ & $2.9 \pm 1.7$ \\
ALG-NS & $135 \pm 49$ & $2.8 \pm 0.8$ \\
CS-F & $145 \pm 17$ & $2.8 \pm 0.9$ \\
\hline
\end{tabular}

the FD into the test solution at $5 \mathrm{~min}$; the amount was 5.7 $\pm 0.4 \mathrm{mg}(38 \pm 3 \%)$ at $30 \mathrm{~min}$. When $0.5 \% \mathrm{CS}$ was added to the base solution, the drug dissolution profile changed. For example, the amount of VST released at $30 \mathrm{~min}$ was increased 1.4-fold compared to the control. The initial dissolution rate was especially accelerated by the addition of $0.5 \% \beta$-CS to film base; the amount of VST dissolved from the FD at $5 \mathrm{~min}$ was $5.3 \pm 0.8 \mathrm{mg}(35 \pm 5 \%)$. However, the addition of $0.5 \%$ chitin to the base solution did not affect the dissolution profile of VST from the FD.

On the other hand, the VST dissolution rate from FDs prepared using Alg-Na was decreased by the addition of ALG-S, as shown in Figure 3. The amount dissolved in the test solution at $30 \mathrm{~min}$ was $2.2 \pm 0.1 \mathrm{mg}$; this is about $15 \%$ of the VST contained in an FD prepared with $1.5 \%$ Alg$\mathrm{Na}$ containing $0.5 \%$ ALG-S. The drug dissolution rate was
TABLE 3: Solubility of VST in physiological saline containing additive at $37^{\circ} \mathrm{C}$.

\begin{tabular}{lc}
\hline Additive & Solubility $(\mathrm{mg} / \mathrm{mL})$ \\
\hline- & 0.16 \\
$0.05 \%$ CS-F & 0.46 \\
$0.10 \%$ CS-F & 0.49 \\
$0.05 \%$ chitin & 0.18 \\
$0.10 \%$ chitin & 0.21 \\
$0.05 \%$ ALG-S & 0.09 \\
$0.10 \%$ ALG-S & 0.05 \\
\hline
\end{tabular}

also reduced in FDs modified with Alg-NS. Likewise, this phenomenon was observed when FDs were prepared with $2 \%$ low-molecular-weight Alg-Na. The VST dissolution from the $\mathrm{FD}$ at $5 \mathrm{~min}$ decreased from $3.6 \pm 0.2 \mathrm{mg}$ to $1.6 \pm 0.2 \mathrm{mg}$ with the addition of $0.5 \%$ ALG-S to the film base.

Table 3 shows the effect of the additives CS or ALG on VST solubility in physiological saline at $37^{\circ} \mathrm{C}$. When a cationic polysaccharide, CS, was added to the test solution, the solubility increased to about 3 times that of the additive-free solution. Conversely, the addition of an anionic polysaccharide, ALG-S, apparently decreased the solubility. In addition, VST solubility remained nearly unchanged by the addition of chitin, an $\mathrm{N}$-acetyl derivative of CS. These 


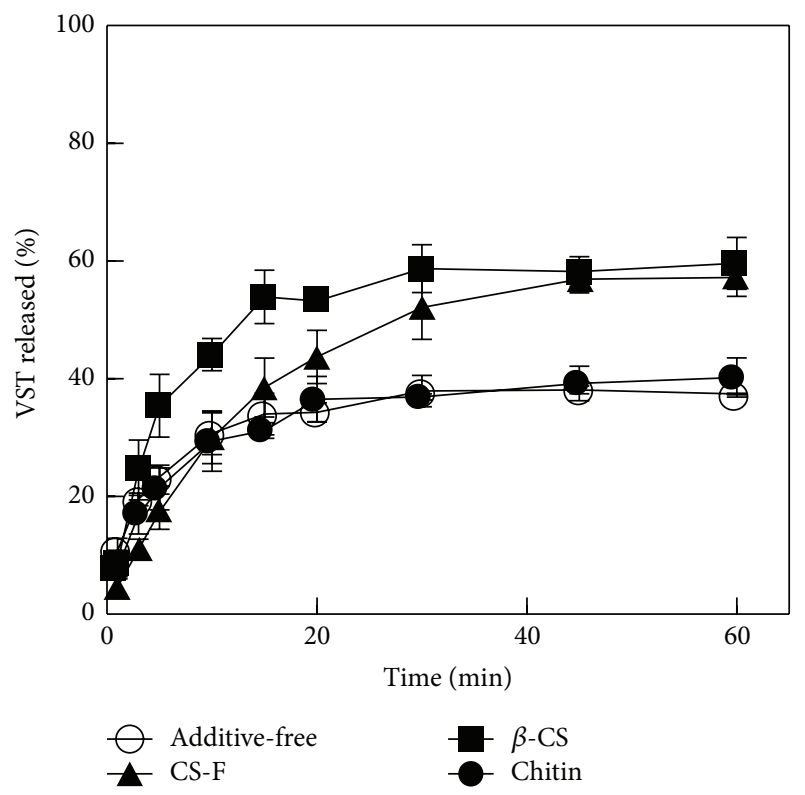

FIGURE 2: Effect of CS (0.5\%) on VST release from FDs prepared with $1.5 \%$ Alg-Na in physiological saline.

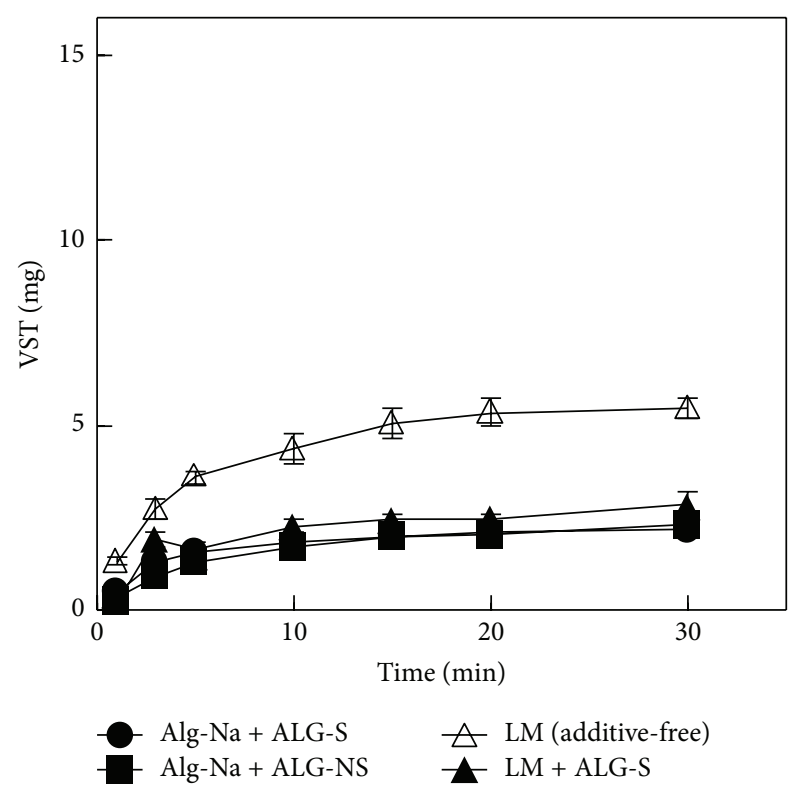

FIGURE 3: Effect of ALG (0.5\%) on VST release from FDs. LM: 2\% low-molecular-weight Alg-Na.

results show that both CS and ALG affect the drug solubility in physiological saline, though these polymers do not dissolve in the medium. VST has two proton dissociating groups, a carboxyl group and a tetrazole, in the structure, and these parts contribute to the aqueous solubility $[12,13]$. Therefore, the change of the drug dissolution rate from the FD may be attributed to an electrostatic interaction between VST and the additive.

Figure 4 shows the effects of the additive ALG-S on the VST dissolution rate from FDs. As the amount of ALG-S

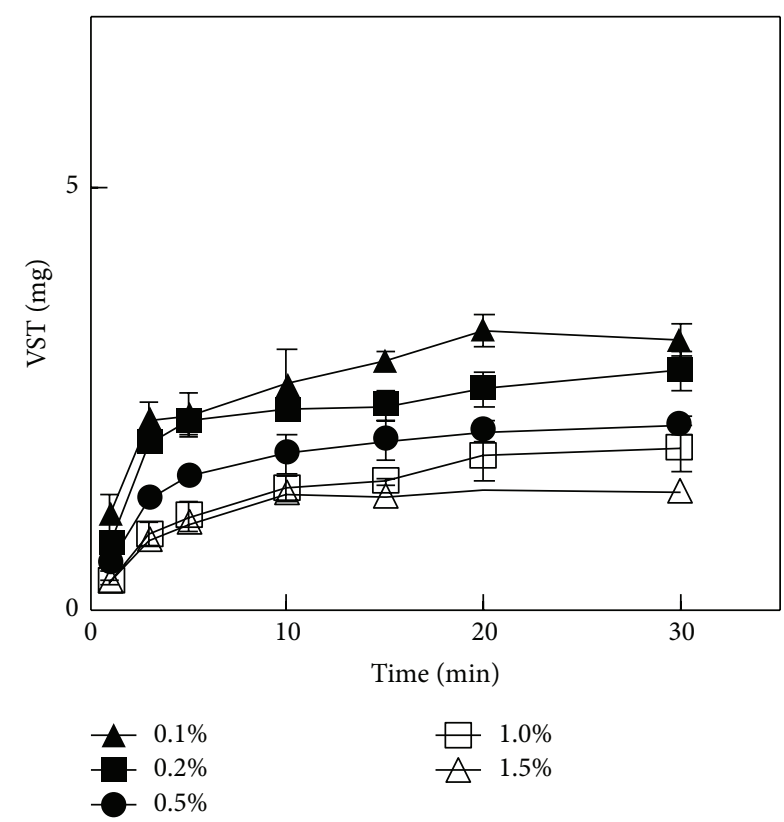

FIGURE 4: Effect of ALG-S concentration on VST release from FDs prepared with $1.5 \%$ Alg-Na.

incorporated into the FD increased, the drug dissolution rate decreased. When $1 \%$ ALG-S was added to the base solution, the amount of VST dissolved at $5 \mathrm{~min}$ was $1.1 \pm 0.2 \mathrm{mg}$, which is about $7 \%$ of the drug incorporated into the FD.

\section{Conclusions}

In this study, FDs were prepared with $1.5-2 \%$ Alg-Na as a film base. The FD immediately disintegrated in a limited dissolution medium and released the VST incorporated into the film matrix. The drug dissolution rate from FDs could be controlled by the addition of CS or ALG to the film base solution. VST is a class II drug in the biopharmaceutical classification system because of its low aqueous solubility; therefore, the dissolution profile in the oral cavity may affect the bioavailability $[14,15]$. FDs prepared with Alg-Na and additives are useful not only for treating localized problems in the oral cavity, but also for simplifying the administration of drugs to patients.

\section{Competing Interests}

The authors declare that they have no competing interests.

\section{Acknowledgments}

This work was supported in part by grants from the OTC SelfMedication Promotion Foundation (2014-2015).

\section{References}

[1] J. C. Visser, W. M. C. Dohmen, W. L. J. Hinrichs, J. Breitkreutz, H. W. Frijlink, and H. J. Woerdenbag, "Quality by 
design approach for optimizing the formulation and physical properties of extemporaneously prepared orodispersible films," International Journal of Pharmaceutics, vol. 485, no. 1-2, pp. 7076, 2015.

[2] M. Preis, C. Woertz, K. Schneider et al., "Design and evaluation of bilayered buccal film preparations for local administration of lidocaine hydrochloride," European Journal of Pharmaceutics and Biopharmaceutics, vol. 86, no. 3, pp. 552-561, 2014.

[3] L. Mazzarino, R. Borsali, and E. Lemos-Senna, "Mucoadhesive films containing chitosan-coated nanoparticles: a new strategy for buccal curcumin release," Journal of Pharmaceutical Sciences, vol. 103, no. 11, pp. 3764-3771, 2014.

[4] H. Okabe, E. Suzuki, Y. Sugiura et al., "Development of an easily swallowed film formulation," International Journal of Pharmaceutics, vol. 355, no. 1-2, pp. 62-66, 2008.

[5] H. Kathpalia and A. Gupte, "An introduction to fast dissolving oral thin film drug delivery systems: a review," Current Drug Delivery, vol. 10, no. 6, pp. 667-684, 2013.

[6] K. B. Liew, Y. T. F. Tan, and K.-K. Peh, "Effect of polymer, plasticizer and filler on orally disintegrating film," Drug Development and Industrial Pharmacy, vol. 40, no. 1, pp. 110-119, 2014.

[7] Y. Murata, T. Isobe, K. Kofuji, N. Nishida, and R. Kamaguchi, "Preparation of fast dissolving films for oral dosage from natural polysaccharides," Materials, vol. 3, no. 8, pp. 4291-4299, 2010.

[8] Y. Murata, K. Kofuji, S. Nakano, and R. Kamaguchi, "Cyclodextrin-modified film dosage forms for oral candidiasis treatment," Pharmacology \& Pharmacy, vol. 6, no. 5, pp. 247-253, 2015.

[9] A. T. Owens, S. C. Brozena, and M. Jessup, "New management strategies in heart failure," Circulation Research, vol. 118, no. 3, pp. 480-495, 2016.

[10] S. S. Imam, A. Ahad, M. Aqil, Y. Sultana, and A. Ali, "A validated RP-HPLC method for simultaneous determination of propranolol and valsartan in bulk drug and gel formulation," Journal of Pharmacy and Bioallied Sciences, vol. 5, no. 1, pp. 6165, 2013.

[11] I. Franceschini, F. Selmin, S. Pagani, P. Minghetti, and F. Cilurzo, "Nanofiller for the mechanical reinforcement of maltodextrins orodispersible films," Carbohydrate Polymers, vol. 136, pp. 676681, 2016.

[12] C. J. Mbah, "Physicochemical properties of valsartan and the effect of ethyl alcohol, propylene glycol and $\mathrm{pH}$ on its solubility," Pharmazie, vol. 60, no. 11, pp. 849-850, 2005.

[13] S. Gora, G. Mustafa, J. K. Sahni, J. Ali, and S. Baboota, "Nanosizing of valsartan by high pressure homogenization to produce dissolution enhanced nanosuspension: pharmacokinetics and pharmacodyanamic study," Drug Delivery, vol. 23, no. 3, pp. 930-940, 2016.

[14] Y.-D. Yan, J. H. Sung, K. K. Kim et al., "Novel valsartanloaded solid dispersion with enhanced bioavailability and no crystalline changes," International Journal of Pharmaceutics, vol. 422, no. 1-2, pp. 202-210, 2012.

[15] W.-J. Xu, H.-J. Xie, Q.-R. Cao et al., "Enhanced dissolution and oral bioavailability of valsartan solid dispersions prepared by a freeze-drying technique using hydrophilic polymers," Drug Delivery, vol. 23, no. 1, pp. 41-48, 2016. 

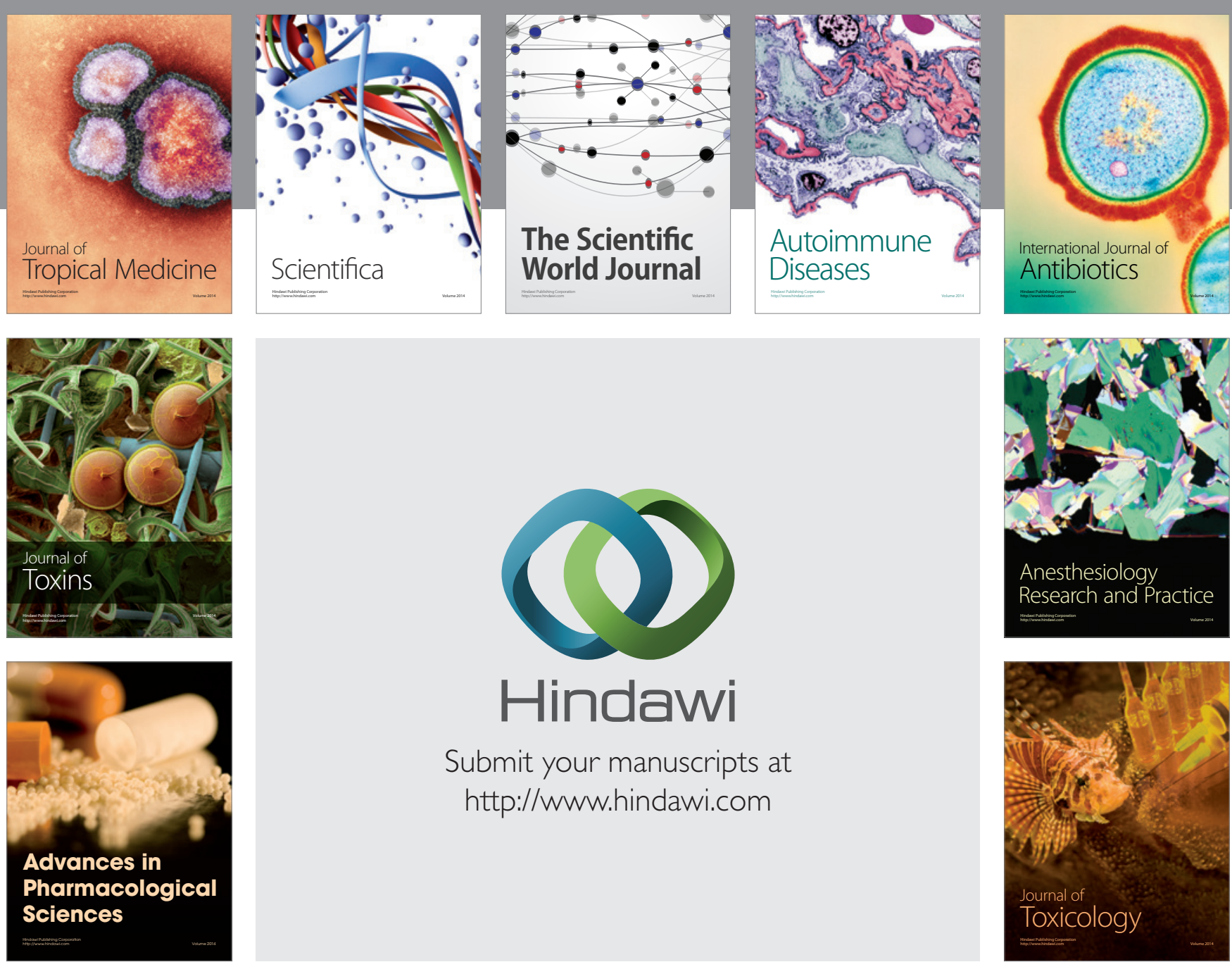

\section{Hindawi}

Submit your manuscripts at

http://www.hindawi.com
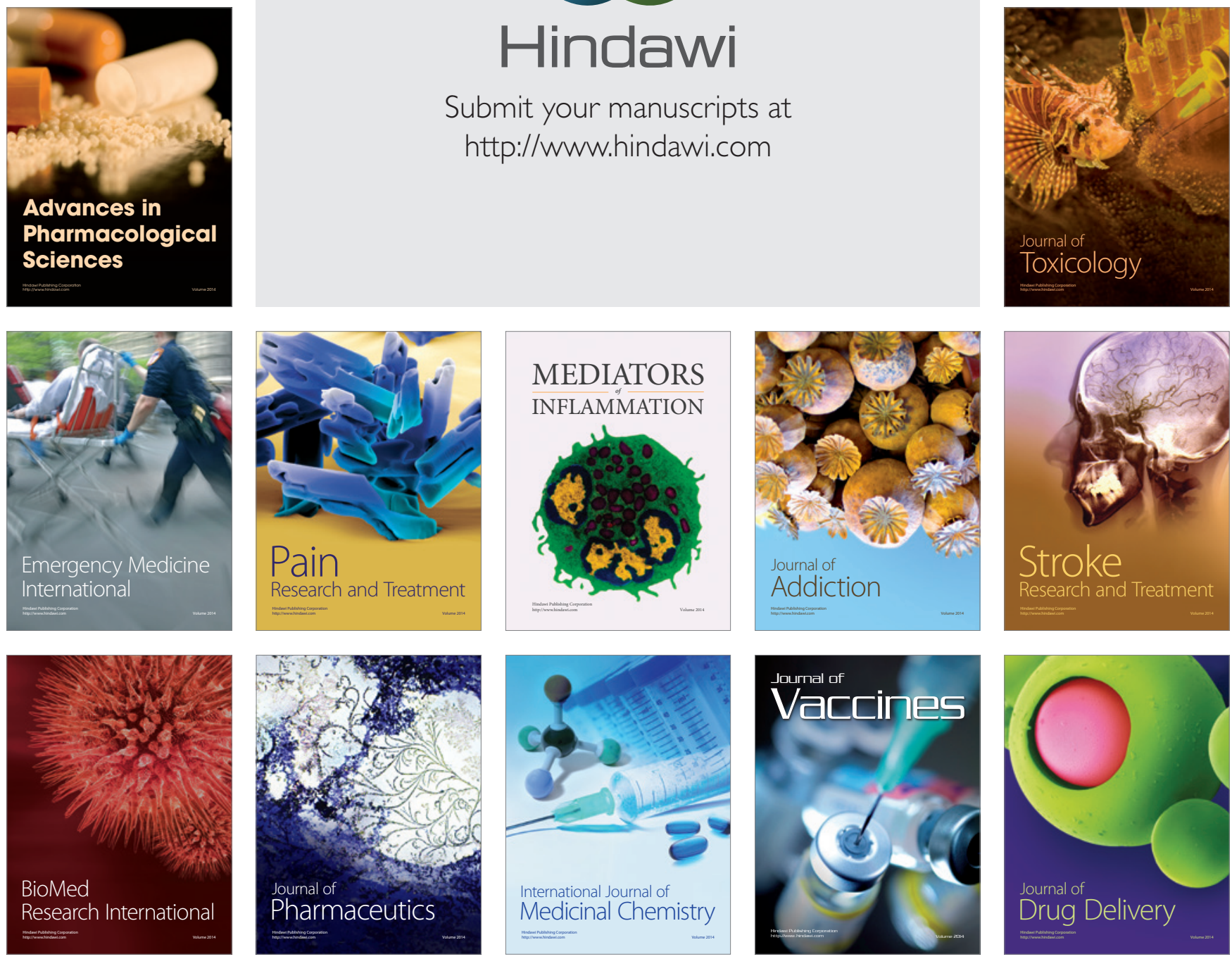